\title{
Simultaneous material and structural optimization by multiscale topology optimization
}

\author{
Raghavendra Sivapuram $^{1}$, Peter D. Dunning ${ }^{2}$ and H. Alicia Kim ${ }^{1,3}$ \\ ${ }^{1}$ Structural Engineering, University of California, San Diego, San Diego, CA 92093, USA \\ ${ }^{2}$ School of Engineering, University of Aberdeen, Aberdeen, UK \\ ${ }^{3}$ School of Engineering, Cardiff University, Cardiff, UK
}

\begin{abstract}
This paper introduces a new approach to multiscale optimization, where design optimization is applied at two scales: the macroscale, where the structure is optimized, and the microscale, where the material is optimized. Thus, structure and material are optimized simultaneously. We approach multiscale design optimization by linearizing and formulating a new way to decompose into macro and microscale design problems in such a way that solving the decomposed problems separately lead to an overall optimum solution. In addition, the macro and microstructural designs are coupled tightly through homogenization and inverse homogenization. This approach is generic in that it allows any number of unique microstructures and can be applied to a wide range of design problems. An advantage of decomposing the problem in this physical way is that it is potentially straight forward to specify additional design requirements at a specific scale or in specific regions of the design domain. The decomposition approach also allows an easy parallelization of the computational methodology and this enables the computational time to be maintained at a practical level. We demonstrate the proposed approach using the level-set topology optimization at both scales, i.e. macrostructural topological design and microstructural topology of architected material. A series of optimization problems, minimizing compliance and compliant mechanism are solved for verification and investigation of potential benefits.
\end{abstract}

Keywords: Multi-scale, topology optimization, level-set method, decomposition, architected material.

\section{Introduction}

The traditional approach to structural design is to select material, then design the structure that best utilizes the given material. Material design, on the other hand, has taken place without understanding of a specific application but for a gross generalization of anticipated or possible needs. The current design practice of structure and material are thus, inherently decoupled and yet the performance and quality of the designs are inextricably linked across the structural and material scales. Recently a new paradigm is being explored where the structure and material are designed simultaneously. Thus, the material properties are tailored to the structural design and vice-versa. This potentially opens up the design space as the material can be directly optimized for the functional needs at the structural scale and can lead to lighter and more efficient structures applicable to many engineering sectors.

Topology optimization is a method that aims to produce optimal structures by optimizing the size, shape and connectivity of the structure (Bendsoe and Sigmund 2004; Deaton and Grandhi 2014). It has been applied to optimize structure and material simultaneously. For example, structural topology and material selection have been simultaneously optimized by designing the material phase (Sigmund 2001; 
Wang and Wang 2004), or the continuous grading between two materials (Dunning et al. 2015; Sigmund and Torquato 1997). Topology optimization has also been combined with the design of layered fiber composite material (De Leon et al. 2012; Lund 2009; Peeters et al. 2014).

Opening up the material design space, topology optimization has been applied at the material microscale (i.e. architected material) in addition to the structural macroscale. A schematic of the multiscale topology optimization is shown in Fig. 1. The hierarchical solution strategy proposed by Rodrigues et al. (2002) allows a unique microstructure to be optimized for each macroscale finite element. Homogenization is used to obtain effective material properties of the microstructures, which are then used to determine the macroscale structural performance. The relative density of a macroscale element is defined from the microscale volume fraction. Thus, if the microstructure becomes completely void, the boundary shape or the topology of the macrostructure is changed. The hierarchical method is similar to the homogenization approach to topology optimization by Bendsoe and Kikuchi (1988) who employed the size and rotation of square or rectangular microscale voids within each macroscale element as the design variables. The difference being that Rodrigues et al. (2002) utilizes the complete topological freedom that is available for optimization at microscale. Although the hierarchical scheme optimizes the topology at two scales, design variables only exist at the microscale. For the multiple load minimum compliance problem, a heuristic scheme based on optimality criteria is employed to update the design variables. This approach allows the microscale optimization problems to be solved separately and parallel computing is exploited. This is important, as the number of design variables can be very large, especially for three dimensional structures (Coelho et al. 2008).

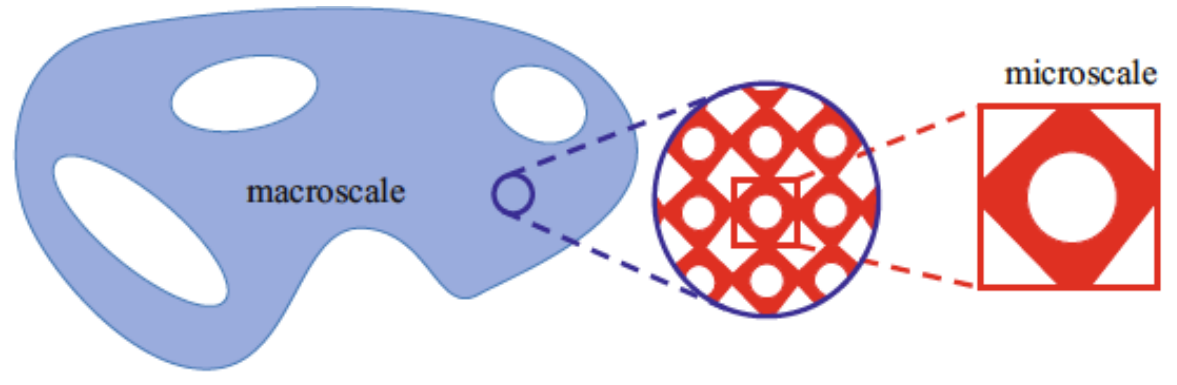

Figure 1. Multi-scale topology optimization.

Another approach was recently proposed by Xia and Breitkopf (2014) where the FE nonlinear multiscale analysis method is used to bridge the scales and a unique microstructure is defined at each element's Gauss integration point. The idea is that the microscale optimization acts as a nonlinear material that adapts to the macroscale strain field. The Bidirectional Evolutionary Structural Optimization (BESO) method is used to optimize the topology at both the macro and microscales. The solution procedure for minimum compliance design starts by computing the macroscale strains. The microscale optimization problems are then to maximize the strain energy density, subject to a volume constraint on the microstructure. These problems are solved independently in parallel. Once all microscale problems converge and the macroscale equilibrium condition is met, the macroscale design variables are updated using sensitivity information that accounts for the material nonlinearity. Again, this 
approach is formulated in the context of the minimization of compliance problem and the application to general problems is not straightforward.

A recent approach proposed by Xia and Breitkopf (2014) uses the $\mathrm{FE}^{2}$ non-linear multi-scale analysis method to bridge the scales and a unique micro-structure is defined at each element Gauss integration point. The idea is that the micro-scale optimization acts as a non-linear material that adapts to the macro-scale strain field. The Bi-directional Evolutionary Structural Optimization (BESO) method is used to optimize the topology at both the macro and micro-scales. The solution procedure for minimum compliance design starts by computing the macro-scale strains. The microscale optimization problems are then to maximize the strain energy density, subject to a volume constraint on the micro-structure. These problems are solved independently using parallel computing. Once all micro-scale problems converge and the macroscale equilibrium condition is met, the macro-scale design variables are updated using sensitivity information that accounts for the material non-linearity. Again, this approach is formulated in the context of the minimization of compliance problem and therefore may not be easily adapted to solve other problems.

An alternative approach to multiscale topology optimization is to consider the macro and microscale designs sequentially (Schury et al. 2012). The first step is to optimize the macrostructure using Free Material Optimization (FMO), where the design variables are the stiffness tensor values for each element. Once the macroscale problem is solved, the second step uses inverse homogenization to design the topology of the microstructures. The microscale problems are to minimize the volume, whilst obtaining the material properties defined from the macroscale optimum. Thus, these problems can be solved in parallel. However, appropriate bounds must be imposed on the stiffness tensor variables in the macroscale problem to ensure that the following microscale problems are feasible. Due to the freedom afforded to the stiffness tensor, setting realistic bounds can present a challenge and some conservatism is required. This compromises the ability of the method to obtain the global optimum. Furthermore, the mass distribution of the structure is not directly considered by the FMO approach, which can be important, for example in dynamic problems.

The multiscale topology optimization methods discussed so far allow the microstructure to vary within the macrostructure. Another formulation is to use the same microstructure throughout the macroscale domain (Liu et al. 2008; Yan et al. 2014). Thus, only one microstructure is optimized and the number of design variables is not significantly greater than a single scale topology optimization. Therefore, the multiscale problem formulation is computationally tractable. Again homogenization is used to bridge the scales and the derivatives of macroscale objective and constraint functions with respect to the microscale design variables are obtained by differentiating the homogenization equations. It is thus straightforward to solve for many structural design problems beyond compliance and has been used to solve for natural frequency (Niu et al. 2009) and multi-objective thermoelastic problems (Deng et al. 2013). However, this approach does not allow local tailoring of material properties and cannot explore the full structure-material design space, thus limiting the potential performance gains that may otherwise be possible. 
In summary, current approaches that use a nonuniform microstructure decompose the problem in some fashion to exploit parallel computing. These decomposition approaches are not easily extendable to a wide range of problems and the existing literature studies focus on the minimization of compliance problem. On the other hand, using a uniform microstructure allows a multiscale solution strategy that is more easily applied to wider range of problems. However, local tailoring of material properties is not possible and limits potential benefits.

The aim of this paper is to develop a generalized framework for multiscale topology optimization that can be used with any number of unique microstructures, including uniform and nonuniform designs. The decomposition strategy introduced in this paper is generally applicable to a range of problems and naturally extends to parallelization, thus the solution strategy is scalable. We use a level-set topology optimization method at both scales, i.e. structural topology and architected material.

The paper is organized as follows. The optimal decomposition of a general multiscale design problem and the computational strategy for solving the coupled multiscale problem, the constraint boundary optimization, the procedure for dealing with inactive microscale domains and microscale constraints are detailed in Section 2. Section 3 discusses the bridging of scales using homogenization for an elastic problem. Section 4 discusses the conventional level-set method which is used to solve each of the decomposed problems. The sensitivity analysis of the multiscale topology optimization problem is detailed in Section 5. Minimum compliance and compliant mechanism examples are presented in Section 6, followed by the conclusions in Section 7.

\section{Multi-scale optimization}

A general multiscale optimization problem can be written as:

$$
\begin{aligned}
\underset{\mathbf{M}, \mathbf{m}_{1}, \mathbf{m}_{2}, \cdots, \mathbf{m}_{N}}{\operatorname{Minimise}} & f\left(\mathbf{M}, \mathbf{m}_{1}, \mathbf{m}_{2}, \cdots, \mathbf{m}_{N}\right) \\
\text { Subject to } & g_{i}\left(\mathbf{M}, \mathbf{m}_{1}, \mathbf{m}_{2}, \cdots, \mathbf{m}_{N}\right) \leq 0, i=1 \cdots N_{g} \\
& \mathbf{M}_{\min } \leq \mathbf{M} \leq \mathbf{M}_{\max } \\
& \mathbf{m}_{j, \min } \leq \mathbf{m}_{j} \leq \mathbf{m}_{j, \max }, j=1 \cdots N
\end{aligned}
$$

where $f$ is the objective function, $g_{i}$ are inequality constraint functions, $N_{g}$ is the number of inequality constraints, $\mathbf{M}$ is the design variable corresponding to the macroscale domain, $\mathbf{m}_{j}$ is the design variable corresponding to the microscale domain $j$ and $N$ is the number of microscale design variables to be optimized. The $\mathbf{M}_{\min }$ and $\mathbf{M}_{\max }$ represent the minimum and maximum bounds for the macroscale design variable $\mathbf{M} . \mathbf{m}_{\mathrm{j}, \text { min }}$ and $\mathbf{m}_{\mathrm{j}, \text { max }}$ are the minimum and maximum bounds for the microscale design variable $\mathbf{m}_{\mathbf{j}}$.

\subsection{Decomposition}

The objective and constraint functions are linearized about the design variables at current iteration $k$ using Taylor's expansion. 


$$
\begin{aligned}
\underset{\Delta \mathbf{M}^{k}, \Delta \mathbf{m}^{k}, \cdots, \Delta \mathbf{m}^{k}{ }_{N}}{\text { Minimise }} & :\left(\frac{\partial f}{\partial \mathbf{M}^{k}}\right) \cdot \Delta \mathbf{M}^{k}+\sum_{j=1}^{N}\left(\frac{\partial f}{\partial \mathbf{m}_{j}^{k}}\right) \cdot \Delta \mathbf{m}_{j}^{k} \\
\text { Subject to } & :\left(\frac{\partial g_{i}}{\partial \mathbf{M}^{k}}\right) \cdot \Delta \mathbf{M}^{k}+\sum_{j=1}^{N}\left(\frac{\partial g_{i}}{\partial \mathbf{m}_{j}^{k}}\right) \cdot \Delta \mathbf{m}_{j}^{k} \leq-g_{i}^{k}, i=1 \cdots N_{g} \\
& \Delta \mathbf{M}^{k}{ }_{\text {min }} \leq \Delta \mathbf{M}^{k} \leq \Delta \mathbf{M}^{k}{ }_{\text {max }} \\
& \Delta \mathbf{m}^{k}{ }_{j, \min } \leq \Delta \mathbf{m}^{k}{ }_{j} \leq \Delta \mathbf{m}^{k}{ }_{j, \max }, j=1 \cdots N
\end{aligned}
$$

where $g_{i}^{k}$ is the value of constraint $i$ at iteration $k$ and $\Delta \mathbf{M}^{k}$ and $\Delta \mathbf{m}_{j}^{k}$ are the design variable update steps for the macroscale design variable $\mathbf{M}^{k}$ and microscale design variable $\mathbf{m}_{\mathrm{j}}^{\mathrm{k}}$ respectively, obtained from iteration $k$.

$$
\begin{aligned}
& \mathbf{M}^{k+1}=\mathbf{M}^{k}+\Delta \mathbf{M}^{k} \\
& \mathbf{m}_{j}{ }^{k+1}=\mathbf{m}_{j}{ }^{k}+\Delta \mathbf{m}_{j}{ }^{k}, j=1 \cdots N
\end{aligned}
$$

In order to exploit parallel computing, the linearized problem (2) is decomposed into $(1+N)$ problems by separating terms associated with the macroscale design variable $\mathbf{M}$, and each of the $N$ microscale design variables, $\mathbf{m}_{j}$ :

$$
\begin{aligned}
& \underset{\Delta \mathbf{M}^{k}}{\operatorname{Minimise}}:\left(\partial f / \partial \mathbf{M}^{k}\right) \Delta \mathbf{M}^{k} \\
& \text { Subject to: }\left(\partial g_{i} / \partial \mathbf{M}^{k}\right) \Delta \mathbf{M}^{k} \leq \gamma_{M, i}, \quad i=1 \cdots N_{g} \\
& \Delta \mathbf{M}^{k}{ }_{\text {min }} \leq \Delta \mathbf{M}^{k} \leq \Delta \mathbf{M}^{k}{ }_{\text {max }} \\
& \left.\begin{array}{c}
\underset{\Delta \mathbf{m}_{j}{ }^{k}}{\operatorname{Minimise}}:\left(\partial f / \partial \mathbf{m}_{j}{ }^{k}\right) \Delta \mathbf{m}_{j}{ }^{k} \\
\text { Subject to }:\left(\partial g_{i} / \partial \mathbf{m}_{j}{ }^{k}\right) \Delta \mathbf{m}_{j}{ }^{k} \leq \gamma_{m, i, j}, i=1 \cdots N_{g} \\
\Delta \mathbf{m}^{k}{ }_{j, \min } \leq \Delta \mathbf{m}^{k}{ }_{j} \leq \Delta \mathbf{m}^{k}{ }_{j, \max }
\end{array}\right\} j=1 \cdots N
\end{aligned}
$$

where $\gamma_{M, i}$ is the $i^{\text {th }}$ constraint boundary corresponding to the macroscale design problem and $\gamma_{m, i j}$ is the $i^{\text {th }}$ constraint boundary corresponding to the $j^{\text {th }}$ microscale design problem.

If the problem is unconstrained $\left(N_{g}=0\right)$, then we can proceed and solve each decomposed problem separately to obtain the updates for the macroscale and microscale design variables. For a constrained problem $\left(N_{g}>0\right)$, the constraint boundaries in the decomposed problems, $\gamma_{M}$ and $\gamma_{m}$ must be compatible with the inequality constraints of the original problem, (1). Thus, the constraint boundaries in the decomposed problems, $(4,5)$ must satisfy the following constraint feasibility condition: 


$$
\begin{aligned}
& \gamma_{M, i}+\sum_{j=1}^{N} \gamma_{m, j, i} \leq-g_{i}^{k} \\
& i=1,2, \cdots N_{g}
\end{aligned}
$$

\subsection{Determination of constraint boundaries}

The search direction for the multiscale problem is defined by the updates for the macroscale and microscale design variables, $\Delta \mathbf{M}$ and $\Delta \mathbf{m}_{j}$. A desirable search direction is one which optimizes the objective whilst meeting the constraints. For the decomposed problems $(4,5)$, the task is to compute the constraint boundaries to achieve such a desirable search direction. The determination of the constraint boundaries is thus, formulated as an optimization problem to minimize the objective function in (2), while satisfying the constraint feasibility condition (6).

$$
\begin{aligned}
& \underset{\gamma_{M}, \gamma_{M}}{\operatorname{Minimise}}: \Delta f^{k}\left(\Delta \mathbf{M}^{k}\left(\gamma_{M}\right), \Delta \mathbf{m}^{k}\left(\gamma_{m}\right)\right)=\left(\frac{\partial f}{\partial \mathbf{M}^{k}}\right) \cdot \Delta \mathbf{M}^{k}\left(\gamma_{M}\right)+\sum_{j=1}^{N}\left(\frac{\partial f}{\partial \mathbf{m}_{j}^{k}}\right) \cdot \Delta \mathbf{m}_{j}^{k}\left(\gamma_{m}\right) \\
& \text { Subject to }: \gamma_{M, i}+\sum_{j=1}^{N} \gamma_{m, j, i} \leq-g_{i}^{k}, i=1 \cdots N_{g} \\
& \gamma_{M, \min } \leq \gamma_{M} \leq \gamma_{M, \max } \\
& \gamma_{m, \text { min }} \leq \gamma_{m} \leq \gamma_{m, \max }
\end{aligned}
$$

where $\gamma_{\mathrm{M} \text {,max }}$ and $\gamma_{\mathrm{M} \text {,min }}$ represent the side limits for constraint boundary vector $\gamma_{\mathrm{M}}$ corresponding to the constraints for the macroscale problem, $\gamma_{\mathrm{m} \text {,max }}$ and $\gamma_{\mathrm{m} \text {,min }}$ are the side limits for the constraint boundary vector $\gamma_{\mathrm{m}}$, corresponding to the constraints for the microscale problems.

The constraint boundary optimization problem of (7) is solved using a nested approach. The objective and constraints are linearized (8). The decomposed problems $(4,5)$ are solved with an initial set of constraint boundaries which meet the constraint feasibility condition. A finite difference method is employed to compute the derivatives of objective function change $\Delta f$, which are then used to update the constraint boundaries.

$$
\begin{gathered}
\underset{\gamma}{\operatorname{Minimise}:}\left(\partial\left(\Delta f^{k}\right) / \partial \gamma_{M}\right)\left(\gamma_{M}^{l+1}-\gamma_{M}^{l}\right)+\left(\partial\left(\Delta f^{k}\right) / \partial \gamma_{m}\right)\left(\gamma_{m}^{l+1}-\gamma_{m}^{l}\right) \\
\text { Subject to }: \gamma_{M, i}+\sum_{j=1}^{N} \gamma_{m, j, i} \leq-g_{i}^{k}, i=1 \cdots N_{g} \\
\gamma_{M, \min } \leq \gamma_{M} \leq \gamma_{M, \max } \\
\gamma_{m, \min } \leq \gamma_{m} \leq \gamma_{m, \max }
\end{gathered}
$$

where $l$ is the iteration number. In practice, a line search strategy is used to compute the constraint boundaries to improve the convergence and stability of the method. If the current objective value of (7) is greater than the previous value, then the constraint boundaries are replaced by: 


$$
\begin{aligned}
& \gamma_{M}^{l}=\omega \cdot \gamma_{M}^{l}+(1-\omega) \cdot \gamma_{M}^{l-1} \\
& \gamma_{m}^{l}=\omega \cdot \gamma_{m}^{l}+(1-\omega) \cdot \gamma_{m}^{l-1}
\end{aligned}
$$

where $\omega$ is a scaling factor, chosen here as 0.5 . Figure 2 shows the pseudo code for solving the constraint boundary optimization problem, where $\eta$ is a small number used in the termination criterion.

$$
\begin{aligned}
& \text { CHOOSE } \gamma_{\mathrm{M}}^{0}, \gamma_{\mathrm{m}}^{0}, \omega, \eta, l=0, \Delta \mathrm{f}\left(\gamma_{\mathrm{M}}^{l-1}, \gamma_{\mathrm{m}}^{l-1}\right)=10^{20} \\
& \text { WHILE max }\left(\left\|\gamma_{\mathrm{M}}^{l}-\gamma_{\mathrm{M}}^{l-1)}\right\|_{\infty},\left\|\gamma_{\mathrm{m}}^{l}-\gamma_{\mathrm{m}}^{l-1}\right\|_{\infty}\right)>\eta \\
& \text { SOLVE decomposed problems }(4,5) \text { for } \mathbf{M}, \mathbf{m}_{\mathrm{j}}{ }^{\prime} \operatorname{susing} \gamma_{\mathrm{M}}, \gamma_{\mathrm{m}} \\
& \text { IS } \Delta \mathrm{f}\left(\gamma_{\mathrm{M}}^{l}, \gamma_{\mathrm{m}}^{l}\right)>\Delta \mathrm{f}\left(\gamma_{\mathrm{M}}^{l-1}, \gamma_{\mathrm{m}}^{l-1}\right) ? \\
& \text { YES : } \boldsymbol{\gamma}_{\mathrm{M}}^{l}=\omega \cdot \gamma_{\mathrm{M}}^{l}+(1-\omega) \cdot \gamma_{\mathrm{M}}^{l-1} \\
& \quad \gamma_{\mathrm{m}}^{l}=\omega \cdot \gamma_{\mathrm{m}}^{l}+(1-\omega) \cdot \gamma_{\mathrm{m}}^{l-1} \\
& \text { NO : COMPUTE } \frac{\partial\left(\Delta \mathrm{f}^{\mathrm{k}}\right)}{\partial \gamma_{\mathrm{M}}^{l}} \cdot\left(\gamma_{\mathrm{M}}^{l+1}-\gamma_{\mathrm{M}}^{l}\right)+\frac{\partial\left(\Delta \mathrm{f}^{\mathrm{k}}\right)}{\partial \gamma_{\mathrm{m}}^{l}} \cdot\left(\gamma_{\mathrm{m}}^{l+1}-\gamma_{\mathrm{m}}^{l}\right) \text { for current } \mathbf{M}, \mathbf{m}_{\mathrm{j}}{ }^{\prime} \mathrm{s} \\
& \quad \operatorname{SOLVE~(8)~for~} \gamma_{\mathrm{M}}, \gamma_{\mathrm{m}} \\
& \quad l=l+1
\end{aligned}
$$

END WHILE

Figure 2. Optimization algorithm for computing constraint boundaries.

The side limits of constraint boundaries in (8) are set such that no constraint boundaries which satisfy the feasibility condition make the decomposed problems infeasible. The maximum and minimum possible constraint changes in each of the decomposed problems for all the constraints, are computed. For example, the maximum and minimum values for the constraint $i$, corresponding to the macroscale problem and the $j^{\text {th }}$ microscale problem are:

$$
\begin{aligned}
& \gamma_{M, i, \min }=\min \left(\frac{\partial g_{i}}{\partial \mathbf{M}} \cdot \Delta \mathbf{M}_{\min }, \frac{\partial g_{i}}{\partial \mathbf{M}} \cdot \Delta \mathbf{M}_{\max }\right) \\
& \gamma_{M, i, \max }=\max \left(\frac{\partial g_{i}}{\partial \mathbf{M}} \cdot \Delta \mathbf{M}_{\min }, \frac{\partial g_{i}}{\partial \mathbf{M}} \cdot \Delta \mathbf{M}_{\max }\right) \\
& \gamma_{m, i, j, \min }=\min \left(\frac{\partial g_{i}}{\partial \mathbf{m}_{j}} \cdot \Delta \mathbf{m}_{j, \min }, \frac{\partial g_{i}}{\partial \mathbf{m}_{j}} \cdot \Delta \mathbf{m}_{j, \max }\right) \\
& \gamma_{m, i, j, \max }=\max \left(\frac{\partial g_{i}}{\partial \mathbf{m}_{j}} \cdot \Delta \mathbf{m}_{j, \min }, \frac{\partial g_{i}}{\partial \mathbf{m}_{j}} \cdot \Delta \mathbf{m}_{j, \max }\right)
\end{aligned}
$$

The maximum and minimum values are then multiplied by a factor, chosen from experience as 0.2 if the problem has a single constraint, or 0.1 for more constraints. To check the feasibility of these values, the decomposed problems $(4,5)$ are solved using the minimum side limits for each constraint. If the problem is infeasible, then the lower limits for the infeasible constraints are multiplied by 0.7 and the problem is solved again. This continues until a feasible solution is obtained. The final values of 
the constraint limits then become the lower side limits for the constraint boundaries in (8).

Once the upper and lower side limits for all constraint boundaries are determined, the initial solution is selected based on the maximum and minimum possible changes for each constraint $\left(\Delta g_{i, \max }\right.$ and $\left.\Delta g_{i, \min }\right)$.

$$
\begin{aligned}
& \Delta g_{i, \text { max }}=\gamma_{M, i, \max }+\sum_{j=1}^{N} \gamma_{m, i, j, \text { max }} \\
& \Delta g_{i, \text { min }}=\gamma_{M, i, \text { min }}+\sum_{j=1}^{N} \gamma_{m, i, j, \min } \quad, i=1 \cdots N_{g}
\end{aligned}
$$

The initial constraint boundaries are then chosen by considering the current value of the constraint $g_{\mathrm{i}}$.

$$
\begin{aligned}
& \gamma_{M, i}^{0}=\left\{\begin{array}{lll}
0.5 \cdot \gamma_{M, i, \min } & , \quad g<0.5 \cdot \Delta g_{i, \text { min }} \\
\left(\gamma_{M, i, \min } \cdot g\right) / \Delta g_{i, \text { min }} & , \quad 0.5 \cdot \Delta g_{i, \text { min }}<g<0 \\
\left(\gamma_{M, i, \max } \cdot g\right) / \Delta g_{i, \text { max }} & , & 0<g<0.5 \cdot \Delta g_{i, \text { max }} \\
0.5 \cdot \gamma_{M, i, \text { max }} & , & 0.5 \cdot \Delta g_{i, \text { max }}<g
\end{array}\right. \\
& \gamma_{m, i, j}^{0}=\left\{\begin{array}{lll}
0.5 \cdot \gamma_{m, i, j, \min } & , \quad g<0.5 \cdot \Delta g_{i, \min } \\
\left(\gamma_{m, i, j, \min } \cdot g\right) / \Delta g_{i, \min } & , \quad 0.5 \cdot \Delta g_{i, \min }<g<0 \\
\left(\gamma_{m, i, j, \max } \cdot g\right) / \Delta g_{i, \text { max }} & , \quad 0<g<0.5 \cdot \Delta g_{i, \max } \\
0.5 \cdot \gamma_{m, i, j, \max } & , & 0.5 \cdot \Delta g_{i, \text { max }}<g
\end{array}\right.
\end{aligned}
$$

where the superscript 0 indicates the initial values. The sum of the initial values of the constraint boundaries define the right hand side of the constraint feasibility condition in $(8)$.

\subsection{Microscale constraints}

This subsection considers a microscale constraint that influences the constraints in other decomposed problem(s). For example, a volume constraint to a microscale domain would need to be reflected on the macroscopic volume. The derivatives for these two volume constraints are directly proportional. This makes one of the constraints redundant, and so is removed from the decomposed problem. We first detect if the derivative of a microscale constraint is proportional to another by computing the following factor.

$$
\alpha=\left(\frac{\partial g}{\partial \mathbf{m}} \cdot \frac{\partial g_{l}}{\partial \mathbf{m}}\right) /\left(\left\|\frac{\partial g}{\partial \mathbf{m}}\right\|_{2} \cdot\left\|\frac{\partial g_{l}}{\partial \mathbf{m}}\right\|_{2}\right)
$$

where $\partial g / \partial \mathbf{m}$ and $\partial g_{l} / \partial \mathbf{m}$ are the derivatives of the constraint $g$ and microscale constraint $g_{l}$ respectively, $\mathbf{m}$ is the design variable of the microscale domain for which $g_{l}$ is defined for. If $\alpha=1$, then the two constraints are directly proportional and if $\alpha=-1$, they are inversely proportional. In both cases the microscale constraint is removed from the decomposed problem and either the upper or lower limit of the 
overall constraint boundary is adjusted to maintain or improve the feasibility of the microscale constraint. For the case $\alpha=1$ the upper limit is adjusted to:

$$
\gamma_{m, g, \max }=\min \left(\gamma_{m, g, \max }, \max \left(g_{l} \cdot\left\|\frac{\partial g}{\partial \mathbf{m}}\right\|_{2} /\left\|\frac{\partial g_{l}}{\partial \mathbf{m}}\right\|_{2}, \gamma_{m, g, \min }\right)\right)
$$

If $\alpha=-1$, then the lower limit is adjusted:

$$
\gamma_{m, g, \min }=\max \left(\gamma_{m, g, \min }, \min \left(-g_{l} \cdot\left\|\frac{\partial g}{\partial \mathbf{m}}\right\|_{2} /\left\|\frac{\partial g_{l}}{\partial \mathbf{m}}\right\|_{2}, \gamma_{m, g, \max }\right)\right)
$$

where $\gamma_{\mathrm{m}, \mathrm{g}, \max }$ and $\gamma_{\mathrm{m}, \mathrm{g}, \min }$ are the maximum and minimum constraint boundaries for the decomposed problem for which $g_{l}$ is defined.

The adjusted limits are used in Eqs. (11) and (12) to define the initial constraint boundaries, where the 0.5 factor is replaced by 1.0 in (12) for a constraint whose derivative is proportional to that of a microscale constraint.

\subsection{Inactive microscale designs}

During optimization, a region of the macroscopic domain may become outside of the design. The microscale design associated with the outside region is no longer considered during optimization and becomes inactive. As the macroscopic design is optimized, its boundaries move which can make these outside regions become a part of the macroscale design again. When a region is reactivated, the microscale design of this region may significantly vary from the neighboring active regions with a discontinuity in material properties. Figure 3 illustrates this: As the macroscale boundary moves ((a) to (b)), the region 4 which has a significantly different microscale design becomes a part of the structure and creates a discontinuity at the interfaces of regions 2 and 3 . In order to reduce the discontinuities, inactive microscale designs are updated at the end of each iteration by copying the microscale design of a neighboring active region, thus maintaining continuity in material properties.

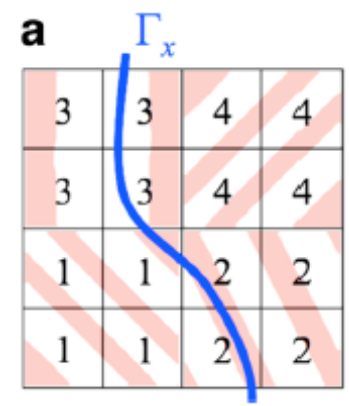

b

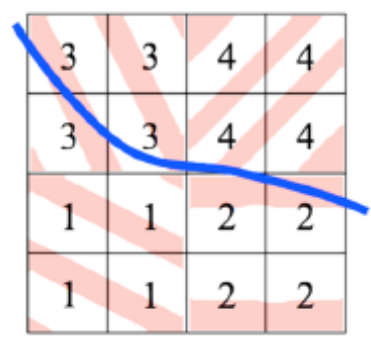

Figure 3. Discontinuity caused by an inactive microscale design a) microscale design 4 is inactive and not optimized b) subsequent iteration where movement of the macroscale boundary makes microscale design 4 active.

\section{Bridging the scales using homogenization}

The asymptotic homogenization method is employed to compute the effective or homogenized properties of a composite material (in this paper, material and voids). The asymptotic expansion of a function is used to solve a differential equation or 
compute the effective properties of a domain. There is a truncation error in the method which is infinitesimal for a small microscopic characteristic length $\zeta$. The asymptotic expansion of a variable $u(\mathrm{x})$ is:

$$
u_{\zeta}(x)=\sum_{i=0}^{\infty} \zeta^{i} u_{i}\left(x, \frac{x}{\zeta}\right)
$$

where $y=x / \zeta$ is called the fast moving variable. The truncation error depends on the number of terms considered on the right hand side and the value of $\zeta$. Allaire (1993) provides the details of the derivation where the coefficients of different powers of $\zeta$ in (16) are used to create a hierarchy of problems to be solved, to compute the homogenized properties.

Here, we outline the procedure to compute the homogenized stiffness tensor to be used in elasticity problems. The classical homogenization (Sanchez-Palencia 1980; Guedes and Kikuchi 1990) with periodic boundary conditions is employed to bridge the scales. The macroscale element is formed by the periodic microscale designs. The microscale design is defined on a unit cell and assumed isotropic properties.

The effective property of the microscale design $\Omega_{\mathrm{m}}$ is computed based on the mechanical behavior of the unit cell. Three unit load cases are applied for 2D problems (uniform extension in two directions and uniform shear) and six unit load cases for 3D problems (three extension and three shear). The effective elasticity tensor for the unit cell is computed as:

$$
E_{p q}^{H}=\int_{\Omega_{m}} E_{p r}\left(\varepsilon_{r}\left(\overline{\mathbf{u}}^{q}\right)-\varepsilon_{r}\left(\mathbf{u}^{q}\right)\right) H(\phi) d \Omega_{m}
$$

where $E_{p q}{ }^{H}$ is the effective (or homogenized) elastic tensor (Voigt notation). $\Omega_{m}$ is the unit cell domain $\left(\left|\Omega_{\mathrm{m}}\right|=1\right)$, $\mathbf{u}^{\mathrm{q}}$ is the characteristic deformations vector for the test case $q, \overline{\mathbf{u}}^{\mathrm{q}}$ is the vector of displacements that produce unit strains over the unit cell (18). $\phi$ is a function which is positive at a point if it lies inside the domain, zero if it lies on the boundary, otherwise negative, and $\mathrm{H}(\phi)$ is the Heaviside function.

$$
\varepsilon_{r}\left(\bar{u}^{q}\right)=\delta_{r q}
$$

where $\delta$ is the Kronecker delta function.

The characteristic deformations $\mathbf{u}^{\mathrm{q}}$ are given as solutions to the periodic cell problem:

$$
\int_{\Omega_{m}}\left(E_{p r} \varepsilon_{r}\left(\overline{\mathbf{u}}^{q}\right)-E_{p r} \varepsilon_{r}\left(\mathbf{u}^{q}\right)\right) \varepsilon_{p}(\mathbf{v}) H(\phi) d \Omega_{m}=0, \forall \mathbf{v} \in \mathrm{V}
$$

where $v$ is $\Omega_{\mathrm{m}}$ - periodic virtual displacement field. Equations (17) and (19) can also be written in compact form (Bendsoe and Kikuchi 1988): 


$$
E_{p q}^{H}=\int_{\Omega_{Y}}\left(E_{r s}\left(\varepsilon_{r}\left(\overline{\mathbf{u}}^{p}\right)-\varepsilon_{r}\left(\mathbf{u}^{p}\right)\right)\left(\varepsilon_{s}\left(\overline{\mathbf{u}}^{q}\right)-\varepsilon_{s}\left(\mathbf{u}^{q}\right)\right)\right) H(\phi) d \Omega_{m}
$$

\section{Level-set topology optimization method}

The conventional level-set method defines a structure using an implicit signed distance function, $\phi(\mathrm{x})$ :

$$
\left\{\begin{array}{l}
\phi(x) \geq 0, x \in \Omega \\
\phi(x)=0, x \in \Gamma \\
\phi(x)<0, x \notin \Omega
\end{array}\right.
$$

where $\Omega$ is the domain of the structure, $\Gamma$ the structure boundary and $x \in \Omega_{\mathrm{d}}$, where $\Omega_{\mathrm{d}}$ is the design domain containing the structure, $\Omega \subset \Omega_{\mathrm{d}}$. The boundary $\Gamma$ is set as the zero level set of the implicit function. The following advection equation is used to update the implicit function and move the position of the boundary:

$$
\frac{\partial \phi(x)}{\partial t}+\nabla \phi(x, t) \frac{d x}{d t}=0
$$

where $t$ represents the level-set evolution time. Since the velocity field of motion by mean curvature is composed of only the normal direction,

$$
\frac{d x}{d t} \cdot \nabla \phi=V_{n} \vec{N} \cdot \nabla \phi=V_{n}|\nabla \phi|
$$

where $V_{n}$ is normal velocity and $\vec{N}$ is the unit outward normal. Discretizing (22) with (23) yields the following update equation (Osher and Sethian 1988):

$$
\phi_{j}^{k+1}=\phi_{j}^{k}-\Delta t\left|\nabla \phi_{j}^{k}\right| V_{n, j}
$$

where $k$ is the iteration number, $j$ is a discrete point in the design domain.

The velocities required for the level set function update in (24) are obtained by solving an optimization problem formulation. We start by a generic single formulation of minimizing an objective function $F(\Omega)$, subject to $N_{g}$ constraints:

$$
\begin{aligned}
& \underset{\Omega}{\text { Minimize: }} F(\Omega)=\int_{\Omega} f Q \\
& \text { Subject to: } G_{i}(\Omega)=\int_{\Omega} g_{i} d \Omega \leq 0, i=1 \cdots N_{g}
\end{aligned}
$$

where $f, g_{i}$ are functions of $x \in \Omega$.

Linearization of the above optimization problem using Taylor's expansion yields: 


$$
\begin{aligned}
& \text { Minimize: } \frac{\partial F}{\partial \Omega^{k}} \cdot \Delta \Omega^{k} \\
& \text { Subject to }: \frac{\partial G_{i}}{\partial \Omega^{k}} \cdot \Delta \Omega^{k} \leq-G_{i}^{k}, i=1 \cdots N_{g}
\end{aligned}
$$

where $\Delta \Omega^{\mathrm{k}}$ is the update for the design domain $\Omega, k$ is the iteration, $G_{i}^{k}$ is the $i^{\text {th }}$ constraint value in the $k^{\text {th }}$ iteration.

The shape sensitivities of the objective and constraint functions can be defined in terms of normal velocities of the boundary points of the domain $\Omega$, which typically take the form of boundary integrals (Allaire et al. 2004; Wang et al. 2003):

$$
\begin{aligned}
& \frac{\partial F}{\partial \Omega} \cdot \Delta \Omega=\Delta t \int_{\Gamma} f \cdot V_{n} d \Gamma \\
& \frac{\partial G_{i}}{\partial \Omega} \cdot \Delta \Omega=\Delta t \int_{\Gamma} g_{i} \cdot V_{n} d \Gamma, i=1 \cdots N_{g}
\end{aligned}
$$

where $\Delta t$ in the case of topology optimization is iteration, $V_{n}$ is the normal velocity of the boundary $\Gamma$. Discretizing the boundary at $n d$ number of points,

$$
\begin{aligned}
& \frac{\partial F}{\partial \Omega} \cdot \Delta \Omega \approx \sum_{j=1}^{n d} \Delta t \cdot V_{n, j}(f \cdot L)_{j}=\mathbf{C}_{\mathbf{f}} \cdot \mathbf{V}_{n} \Delta t \\
& \frac{\partial F}{\partial \Omega} \cdot \Delta \Omega \approx \sum_{j=1}^{n d} \Delta t \cdot V_{n, j}\left(g_{i} \cdot L\right)_{j}=\mathbf{C}_{i} \cdot \mathbf{V}_{n} \Delta t, i=1 \cdots N_{g}
\end{aligned}
$$

where $L_{j}$ is the discrete length of the boundary (or surface area in 3D) around a discrete boundary point $j, \mathbf{C}_{\mathrm{f}}$ and $\mathbf{C}_{\mathrm{i}}$ are the vectors containing integral coefficients, $V_{n}$ is the vector of normal velocities at the $n d$ discrete boundary points. For a constrained problem, we can write $V_{n} \Delta t=\alpha \mathbf{d}$ where $\alpha \neq 0$ is the distance travelled along the search direction $\mathbf{d}$ for the level-set boundary update. Thus, $\mathbf{d}$ being a unit vector is to be added as an equality constraint. Expressing $V_{n}$ as a function of $\alpha$ and $\mathbf{d}$, the optimization problem (26) can be written as:

$$
\begin{aligned}
& \underset{\alpha^{k}, \mathbf{d}^{k}}{\operatorname{Minimize}}: \Delta t \mathbf{C}_{f}^{k} \cdot \mathbf{V}_{n}^{k}\left(\alpha^{k}, d^{k}\right) \\
& \text { Subject to : } \Delta t \mathbf{C}_{i}^{k} \cdot \mathbf{V}_{n}^{k}\left(\alpha^{k}, \mathbf{d}^{k}\right) \leq-g_{i}^{k}, i=1 \cdots N_{g} \\
& \left(\mathbf{d}^{k}\right)^{\top}\left(\mathbf{d}^{k}\right)=1 \\
& \mathbf{V}_{n, \text { min }}^{k} \leq \mathbf{V}_{n}^{k} \leq \mathbf{V}_{n, \text { max }}^{k}
\end{aligned}
$$

The Lagrangian function for (29) is given by:

$$
L\left(\alpha^{k}, \mathbf{d}^{k}, \lambda^{k}, \mu^{k}\right)=\alpha^{k} \mathbf{C}_{f}^{k} \cdot \mathbf{d}^{k}+\sum_{i=1}^{N_{g}} \lambda_{i}^{k}\left(\alpha^{k} \mathbf{C}_{i}^{k} \cdot \mathbf{d}^{k}+g_{i}^{k}\right)+\mu^{k}\left(\mathbf{d}^{k}\right)^{\top}\left(\mathbf{d}^{k}\right)-1
$$


where $\lambda^{\mathrm{k}}$ is the vector of Lagrange multipliers for the $N_{g}$ inequality constraints, $\mu^{k}$ is the Lagrange multiplier for the equality constraint in Eq. (29) at iteration $k$. We know that for $\mathbf{d}$ be the solution of the optimization problem in (29), itis necessary that the first order optimality condition in (31) of the Lagrangian (30) is satisfied.

$$
\frac{\partial L}{\partial \mathbf{d}^{k}}=\alpha^{k} \mathbf{C}_{f}^{k}+\alpha^{k} \sum_{i=1}^{N_{g}} \lambda_{i}^{k} \mathbf{C}_{i}^{k}+2 \mu^{k} \mathbf{d}^{k}=0
$$

Solving (31) for $\mathbf{d}^{\mathrm{k}}$ and substituting in the equality constraint of (29), we get:

$$
\mathbf{d}^{k}=\frac{\mathbf{C}_{f}^{k}+\sum_{i=1}^{N_{g}} \lambda_{i}^{k} \mathbf{C}_{i}^{k}}{\left\|\mathbf{C}_{f}^{k}+\sum_{i=1}^{N_{g}} \lambda_{i}^{k} \mathbf{C}_{i}^{k}\right\|}
$$

Substituting (32) into (29) eliminates the equality constraint and the optimization problem becomes:

$$
\begin{aligned}
& \underset{\alpha^{k}, \lambda^{k}}{\operatorname{Minimize}}: \Delta t \mathbf{C}_{f}^{k} \cdot \mathbf{V}_{n}^{k}\left(\alpha^{k}, \lambda^{k}\right) \\
& \text { Subject to }: \Delta t \mathbf{C}_{i}^{k} \cdot \mathbf{V}_{n}^{k}\left(\alpha^{k}, \lambda^{k}\right) \leq-g_{i}^{k}, i=1 \cdots N_{g} \\
& \mathbf{V}_{n, \text { min }}^{k} \leq \mathbf{V}_{n}^{k} \leq \mathbf{V}_{n, \text { max }}^{k}
\end{aligned}
$$

The velocities $V_{n, j}$ are determined by solving (33) for $\alpha^{\mathrm{k}}, \lambda^{\mathrm{k}}$ using sequential quadratic programming in NLOPT (Johnson 2014) and substituted into (24) to obtain the new boundary.

In this work, as topology optimization is applied to the decomposed formulation, each decomposed problem can be considered as a single scale optimization problem.

\section{Sensitivity Analysis}

The multiscale optimization formulation introduced in Section 2 can be considered a general framework for a range of multiscale problems. This section presents the sensitivity analysis to apply level-set topology optimization of Section 4 to multiscale optimization of Section 2. This enables simultaneous optimization of the topologies of the architected material and the macroscopic structure.

The general multiscale optimization problem of (1) can be rewritten for elasticity problems as:

$$
\begin{aligned}
\underset{\mathbf{M}, \mathbf{m}_{1}, \cdots, \mathbf{m}_{N}}{\operatorname{Minimise}}: & f\left(\mathbf{M}, \mathbf{E}^{H}\left(\mathbf{m}_{1}\right), \mathbf{E}^{H}\left(\mathbf{m}_{2}\right), \cdots, \mathbf{E}^{H}\left(\mathbf{m}_{N}\right)\right) \\
\text { Subject to }: & g_{i}\left(\mathbf{M}, \mathbf{E}^{H}\left(\mathbf{m}_{1}\right), \mathbf{E}^{H}\left(\mathbf{m}_{2}\right), \cdots, \mathbf{E}^{H}\left(\mathbf{m}_{N}\right)\right) \leq 0, i=1 \cdots N_{g} \\
& \mathbf{M} \subset \Omega_{d} \\
& \mathbf{m}_{j} \subset \Omega_{m}, \quad j=1 \cdots N
\end{aligned}
$$


There are $(1+N)$ design domains, one for the macroscale and $N$ for the microscale. It is assumed that the user would define the number $N$ and the spatial distribution of the unique material regions. $N=1$ specifies uniform structured material throughout the macrostructure and the maximum value $N$ is the number of elements in the macroscale domain. Any number between 1 and the total number of macroscopic elements $N$, and any spatial distributions of microstructures are possible. The multiscale formulation decomposes to consider optimization of the macroscopic domain and each microscopic unit cell domain. The effective elasticity tensor of the microstructure is computed using (20).

To determine the velocity in (33), the integral coefficients for the objective and constraints are required in each problem. The shape sensitivities for the macroscale are obtained by considering that the material properties are defined from the current microscale designs (20). The shape sensitivities for the microscales are obtained using the macroscale FE discretization and the chain rule. The objective function is a function of effective stiffness which in turn is a function of microscopic topology as follows:

$$
f\left(\mathbf{K}\left(\mathbf{M}, \mathbf{E}^{H}\left(\mathbf{m}_{1}\right), \mathbf{E}^{H}\left(\mathbf{m}_{2}\right), \cdots, \mathbf{E}^{H}\left(\mathbf{m}_{N}\right)\right)\right)
$$

where the macroscale stiffness matrix, $\mathbf{K}$, is the assembly of element stiffness matrices:

$$
\mathbf{K}=\mathrm{A}_{e=1}^{N_{e}} \mathbf{k}_{e}\left(\mathbf{E}^{H, e}\right), \quad \mathbf{k}_{e}=\sum_{n=1}^{n g} \mathbf{B}_{n}^{T} \cdot \mathbf{E}^{H, e} \cdot \mathbf{B}_{n} \cdot z_{n}
$$

where $\mathbf{K}$ is the macroscale stiffness matrix, $\mathbf{k}_{\mathrm{e}}$ is a macroscale element stiffness matrix, $\mathbf{B}$ is the strain-displacement matrix, $z_{n}$ the integration weight for integration point $n, n g$ the number of integration points, $\mathbf{E}^{\mathrm{H}, \mathrm{e}}$ is the effective elasticity tensor for a microstructure $e$. A is the assembly operator.

The shape sensitivities for the homogenized elastic tensor and effective density are:

$$
\frac{\partial E_{p q}^{H, j}}{\partial \mathbf{m}_{j}} \cdot \Delta \mathbf{m}_{j}\left(V_{n, j}\right)=\Delta t \int_{\Gamma_{j}}\left(E_{r s}\left(\varepsilon_{r}\left(\overline{\mathbf{u}}^{p}\right)-\varepsilon_{r}\left(\mathbf{u}^{p}\right)\right)\left(\varepsilon_{s}\left(\overline{\mathbf{u}}^{q}\right)-\varepsilon_{s}\left(\mathbf{u}^{q}\right)\right)\right) V_{n, j} d \Gamma_{j}
$$

where $\mathbf{m}_{\mathrm{j}}$ and $\Gamma_{\mathrm{j}}$ are the domain and boundary of the $j^{\text {th }}$ microdesign, such that $\mathbf{m}_{\mathrm{j}} \subset \Omega_{\mathrm{m}}, V_{n, j}$ is the normal velocity of the boundary of the domain $\mathbf{m}_{\mathrm{j}}$.

The derivative of $\mathbf{K}$ with respect to an entry of $\mathbf{E}^{\mathrm{H}, \mathrm{j}}$ is

$$
\frac{\partial \mathbf{K}}{\partial E_{p q}^{H, j}}={\underset{A}{e=1}}_{N_{e}} \delta_{j, e} \cdot\left[\sum_{n=1}^{n g} \mathbf{B}_{n}{ }^{T} \cdot \frac{\partial \mathbf{E}^{H, j}}{\partial E_{p q}^{H, j}} \cdot \mathbf{B}_{n} \cdot z_{n}\right]
$$


The derivative in (38) is then substituted into the derivative of $f$ in (35) with respect to an entry in $\mathbf{E}^{\mathrm{H}, \mathrm{j}}$. The chain rule is then used to compute the shape sensitivity of $f$ with respect to the $j^{\text {th }}$ microstructure:

$$
\frac{\partial f}{\partial \mathbf{m}_{j}} \cdot \Delta \mathbf{m}_{j}\left(V_{n, j}\right)=\sum_{p=1}^{s} \sum_{q=1}^{s} \frac{\partial f}{\partial E_{p q}^{H, j}} \cdot \frac{\partial E_{p q}^{H, j}}{\partial \mathbf{m}_{j}} \cdot \Delta \mathbf{m}_{j}\left(V_{n, j}\right)
$$

where $s$ is three for 2D problem, six for 3D problem. Now, substituting the effective elastic property shape sensitivities (37) into (39) and rearranging, the microscale shape sensitivity is obtained:

$$
\frac{\partial f}{\partial \mathbf{m}_{j}} \cdot \Delta \mathbf{m}_{j}\left(V_{n, j}\right)=\Delta t \int_{\Gamma_{j}}\left[\sum_{p=1}^{s} \sum_{q=1}^{s} \frac{\partial f}{\partial E_{p q}^{H, j}} \cdot\left(E_{r s}\left(\varepsilon_{r}\left(\overline{\mathbf{u}}^{p}\right)-\varepsilon_{r}\left(\mathbf{u}^{p}\right)\right)\left(\varepsilon_{s}\left(\overline{\mathbf{u}}^{q}\right)-\varepsilon_{s}\left(\mathbf{u}^{q}\right)\right)\right)\right] \cdot V_{n, j} d \Gamma_{j}
$$

For a general objective function, its derivatives with respect to each entry in the elastic property matrix (39) is to be computed. These derivatives are then used to combine the shape sensitivities for the effective elasticity tensor at the microscale to arrive at a single shape sensitivity for the objective function with respect to a change in the microstructure (40).

The multiscale level-set topology optimization algorithm is summarized below:

1. Compute the effective stiffness tensor (20) for each microstructure.

2. Assemble the macroscale FE matrices (36).

3. Solve the macroscale governing equations.

4. Check for convergence. Otherwise proceed.

5. Compute the shape sensitivities (40).

6. Calculate the constraint boundaries using the constraint boundary optimization mentioned in Fig. 2.

7. Determine the velocity (33).

8. Update the macro and microscale level-set functions, (24).

Without parallel computing the solution time of the above algorithm increases approximately linearly with the number of microstructures, primarily due to steps 1,5 , 6, 7 and 8 being performed over each microstructure. However, due to the decomposition, these steps can all be performed in parallel, thus making the proposed multiscale topology optimization method computationally tractable.

\section{Examples}

The multiscale topology optimization method is demonstrated using several examples. In all examples, four-node bilinear plane stress elements are used to discretize the macro and microscale design domains.

\subsection{A deep beam rested on two supports}

The first example is a deep beam resting on two supports, with a vertical load applied along the top edge as shown in Fig. 4. This example is taken from Jog et al. (1994) where each element has a different microstructure made of rank-2 layered composites. 
The design variables used were the averaged density of the stiff layer in the rank-1 composite, the averaged density of the stiff material in the rank-2 composite, and the angle of inclination of the material coordinate system with the global reference frame.

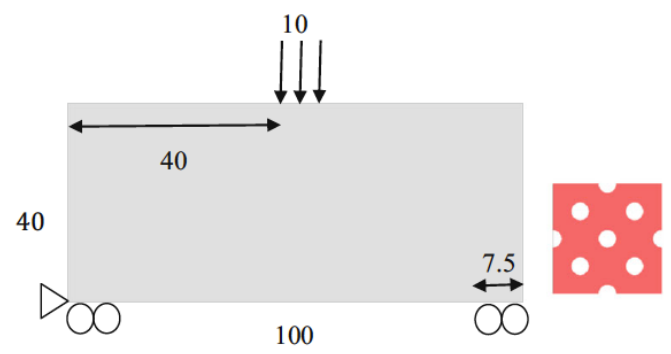

Figure 4. Initial designs of macrostructure and microstructure.

The multiscale method in this paper is applied to solve this conventional homogenization problem. The macroscale optimization is switched off, and every element is allowed to have its own microstructure by topology optimization in each of the microstructures. The bridging between the macro and micro scales is done using homogenization discussed in Section 3. The optimization method essentially becomes the same as the classic homogenization topology optimization approach with the key difference being the material microstructures where the present method allows the design freedom of topology optimization and the classic homogenization approach optimizes rank-2 composites.

Taking advantage of symmetry, only right half of the problem is solved with a $50 \times$ 40 mesh. Each of the 2,000 elements are associated with a unique microstructure. The macroscopic volume constraint is set to $60 \%$.

The optimized solution is shown in Fig. 5 using the plot of elemental porosity of the domain. The solution agrees well with that obtained by Jog et al. (1994), with macroscale elements of high density connecting the load to the boundary conditions. The checkerboard pattern in the center section is also seen in the rank-2 composite homogenization solution of Jog et al. (1994), as commonly seen in this type of optimization without filtering. The density distribution of the solutions including the checkerboard regions are comparable. This verifies the coupling of macromicrostructural topology optimization using the level set method via homogenization.

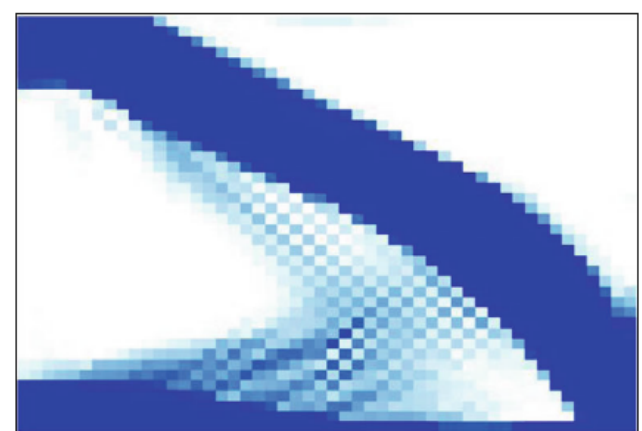

Figure 5. Elemental Porosity plot - Optimum solution for the deep beam.

\subsection{Simply supported beam}

A simply supported beam of aspect ratio 4 with a vertical load at the center, is optimized with the initial macroscopic structure (Fig. 6a), and a uniform material 
microstructure (Fig. 6b). Due to symmetry, only the right half of the beam is optimized and the macroscale domain is discretized using $160 \times 80$ elements. The unit cell for the microscale domain is discretized using $100 \times 100$ elements, Fig. $6 \mathrm{~b}$. The Young's modulus is $2.1 \times 10^{5}$ and Poisson's ratio 0.3 . This example is taken from Liu et al. (2008) for verification.

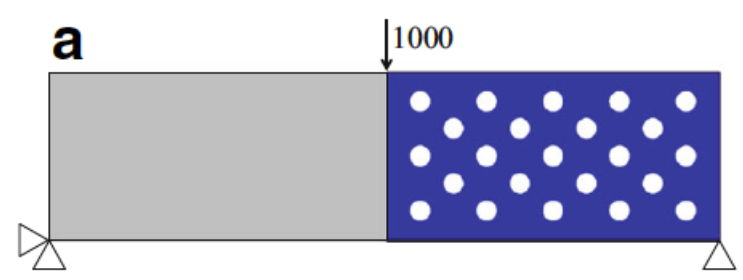

b

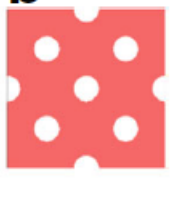

Figure 6. Simply supported beam: a) macroscale initial design, loading and boundary conditions b) microscale initial design.

The objective is to minimize compliance, subject to a volume constraint at the macroscale and a microscale volume constraint at the microscale. Global volume constraints are defined as a percentage of macroscale design domain, but are also affected by the microstructures. The global volume of the multiscale design is defined as:

$$
\text { Volume }=\int_{\Omega_{d}} H(\phi) \cdot\left(\rho^{H} / \rho\right) d \Omega_{d}
$$

where $\rho^{H}$ is the homogenized value of the density (42), which can vary over the macroscale domain according to the mapping of microscale designs to macroscale elements. A microscale volume constraint is applied only to the microstructures and defined as a percentage of microscale design domain. The effective density, $\rho^{H}$ of the microstructure is simply computed as:

$$
\rho^{H}=\int_{\Omega_{Y}} \rho \cdot H(\phi) d \Omega_{Y}
$$

where $\rho$ is the density of the isotropic material.

First we optimize the multiscale problem with a range of macroscopic volume constraints and a fixed microstructure volume constraint of $40 \%$. The results show that as the macroscopic volume fraction increases, compliance decreases, Table 1. This is because, for more material available, the optimization algorithm can produce a stiffer structure. This essentially only influences the macroscopic structures which add more members as more material is available. The optimum microstructure is the same for all cases, with horizontal stiffeners and a diagonal lattice, where one diagonal is slightly thicker than the other. The topological solutions at macro and microscales are comparable with those obtained by Liu et al. (2008). 
Table 1. Simply support beam results with a $40 \%$ microscale volume constraint.

\begin{tabular}{|l|c|c|c|}
\hline $\begin{array}{c}\text { Volume } \\
\text { fraction }\end{array}$ & Macrostructure & $\begin{array}{c}\text { Microstructure } \\
(\mathbf{4 0 \%} \text { Volume })\end{array}$ & Compliance \\
\hline $7.50 \%$ & & 1072 \\
\hline $9.00 \%$ & & \\
\hline $12.00 \%$ & & \\
\hline
\end{tabular}

For the second series of optimization, the global volume constraint is fixed at $12 \%$ and the structure optimized with different microscale volume constraints for microstrucutres. These solutions are compared with two further runs, one with no microscale constraint and the other using single scale optimization with solid isotropic material. The results are summarized in Table 2 which show that as the microstructure volume constraint is increased, compliance decreases. In the case of $100 \%$ microstructure volume constraint, the optimum microstructure is solid. This is consistent with the trend and the difference in the objective function between this result (339) and a single mascroscale level set topology optimization result (336) is less than $1 \%$, i.e. negligible. The microstructure experiences a range of different strains from all the macroscale elements, with different principal strain directions. This result is not surprising, as the objective is to minimize compliance, or maximize the stiffness of the beam, the optimization procedure makes use of more material to stiffen the structure, so as to resist the loads. Thus, for minimum compliance design, porous material is not optimal. When a microscale volume constraint is enforced for the porous material, the microscale design has a diagonal lattice, with horizontal members at the top and bottom. 
Table 2. Simply supported beam results with $12 \%$ global volume constraint.

\begin{tabular}{|c|c|c|c|}
\hline Macrostructure & Microstructure & $\begin{array}{c}\text { Volume } \\
\text { fraction }\end{array}$ & Compliance \\
\hline & & $30 \%$ & 837 \\
\hline & & $40 \%$ & 702 \\
\hline & & $50 \%$ & 607 \\
\hline & & $100 \%$ & 339 \\
\hline & & & \\
\hline
\end{tabular}

\section{$6.3 \mathrm{~L}$ beam}

This example investigates multiscale topology optimization with nonuniform microstructure. The objective is to minimize the compliance of an L shaped beam, Fig. $7 \mathrm{a}$, subject to a global volume constraint of $18 \%$. This is solved with and without a microscale volume constraint of $40 \%$. Both problems are solved three times each using a different number of microstructures: one (uniform), three and 12 microsctructures. The mapping for three and 12 microstructures is shown in Fig. 8. The macroscale domain is discretized by unit sized elements. The microscale unit cell is discretized using $50 \times 50$ mesh and the initial design for all microstructures is shown in Fig. 7b. The material properties of the isotropic material are Young's modulus of 100 and Poisson's ratio of 0.3 .

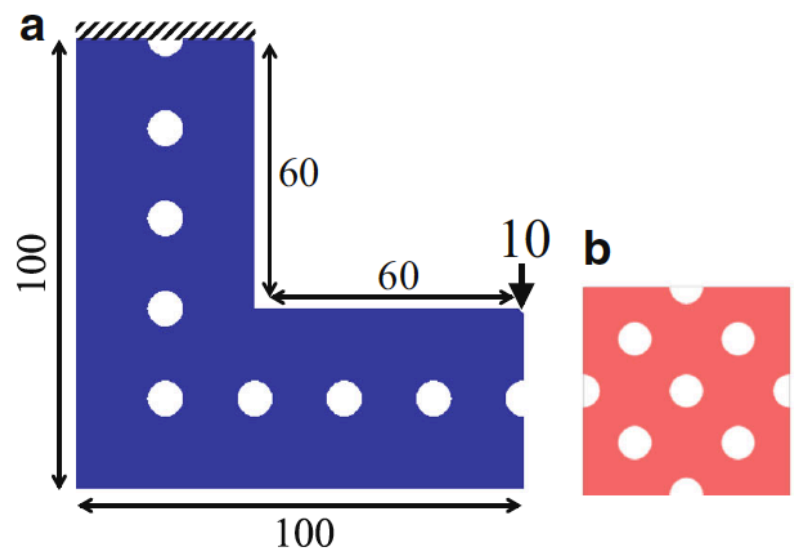

Figure 7. L beam: a) macroscale initial design, loading and boundary conditions, b) initial microscale design. 


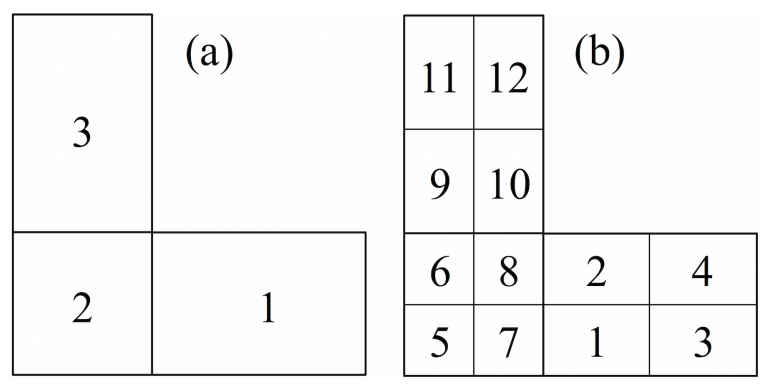

Figure 8. L beam mapping of microscale designs to macro-structure elements, a) 3 microstructures, b) 12 microstructures.

The solutions obtained using a microscale volume constraint show that as the number of unique microstructures increases, the compliance decreases, Table 3. This is expected as the increasing number of microstructures expands the scope for the material tailoring, hence the available design space. The solutions with microscale volume constraints are shown in Figs. 9, 10 and 11. The solution using 12 microstructures shows evidence that the macrostructure adapts to the microscale designs and vice-versa, where the macroscopic structural members are aligned with the edges of microstructure regions, Fig. 11. This leads to a more consistent principal strain direction for the set of macroscale elements associated with a single microstructure, which in turn enables a more tailored microscale designs, such as the unidirectional microstructural designs. Shear resistant microstructures with diagonal members emerge in regions where the load distributions are shear dominant. This demonstrates the coupling of the optimum macro and microstructures. The solutions are dependent on the number and spatial specification of allowable microstructures. When porous materials are required, increasing the number of allowable microstructures leads to a solution with an improved objective.

When the microstructure volume constraint is removed and the multiscale optimizer is let to determine the optimal microscale volume, all optimal macroscopic structures are similar, Fig. 12, and their compliance values are within 1\%, Table 3. Their microstructural designs are solid. This suggests that the optimum volume fraction for a local microstructure is $100 \%$. The compliance with solid microstructure is lower than the best design obtained using microscale volume constraints $<100 \%$ (Table 3 ), which suggests again that the optimal microstructure for a single load compliance problem is solid material.

Table 3. L beam compliance values.

\begin{tabular}{|r|c|c|}
\hline \multirow{2}{*}{$\begin{array}{l}\text { No. micro- } \\
\text { structures }\end{array}$} & \multicolumn{2}{|c|}{ Compliance } \\
\cline { 2 - 3 } & $\begin{array}{l}\text { 40\% microscale } \\
\text { volume constraint }\end{array}$ & $\begin{array}{l}\text { No microscale } \\
\text { volume constraint }\end{array}$ \\
\hline 1 & 905.7 & 374.3 \\
\hline 3 & 712.8 & 374.0 \\
\hline 12 & 642.9 & 372.6 \\
\hline
\end{tabular}




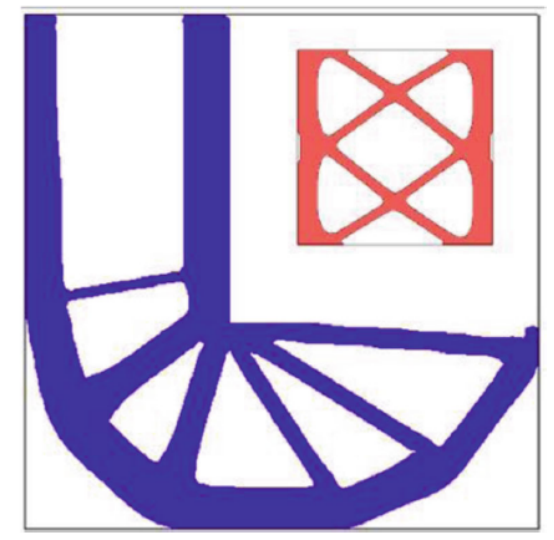

Figure 9. $\mathrm{L}$ beam solution with uniform microstructure and $\mathbf{4 0 \%}$ local volume constraint.

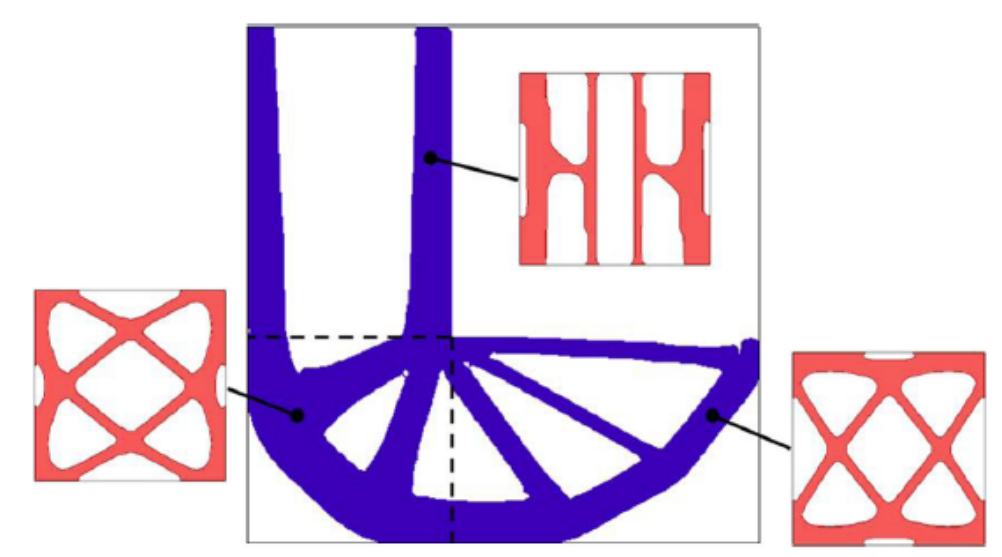

Figure 10. $L$ beam solution with three microstructures and $40 \%$ local volume constraints.

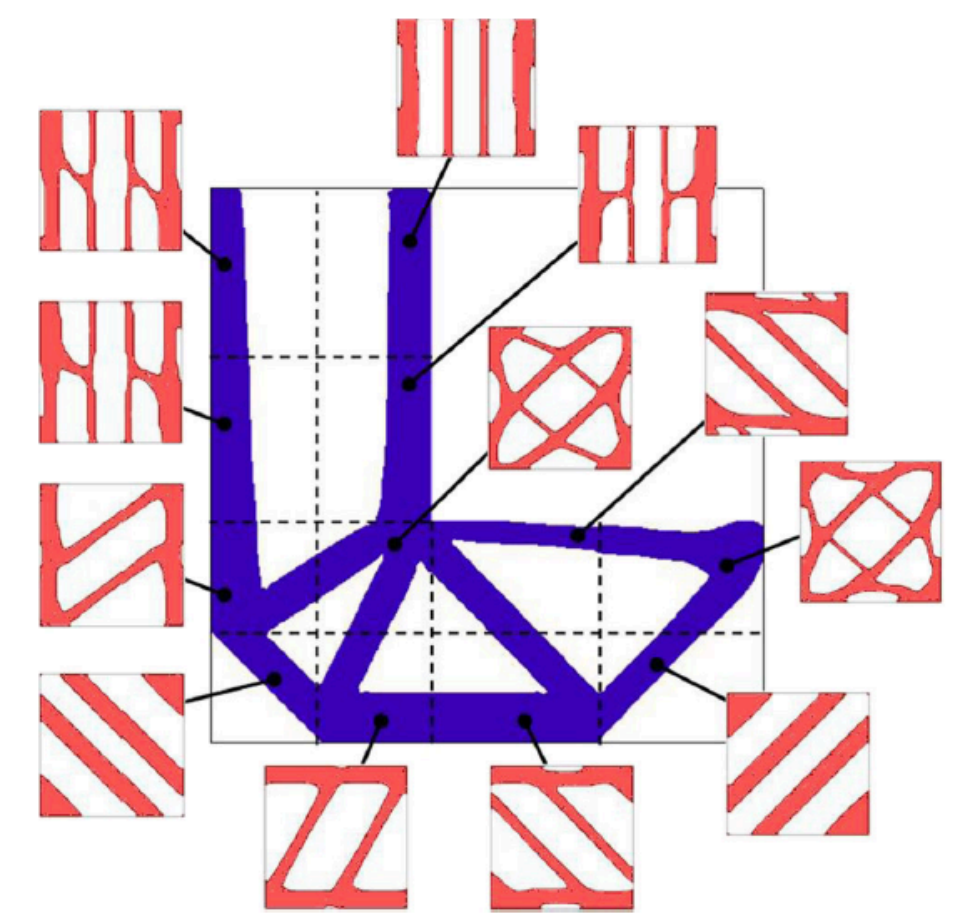

Figure 11. L beam solution with 12 micro-structures and $40 \%$ local volume constraints. 


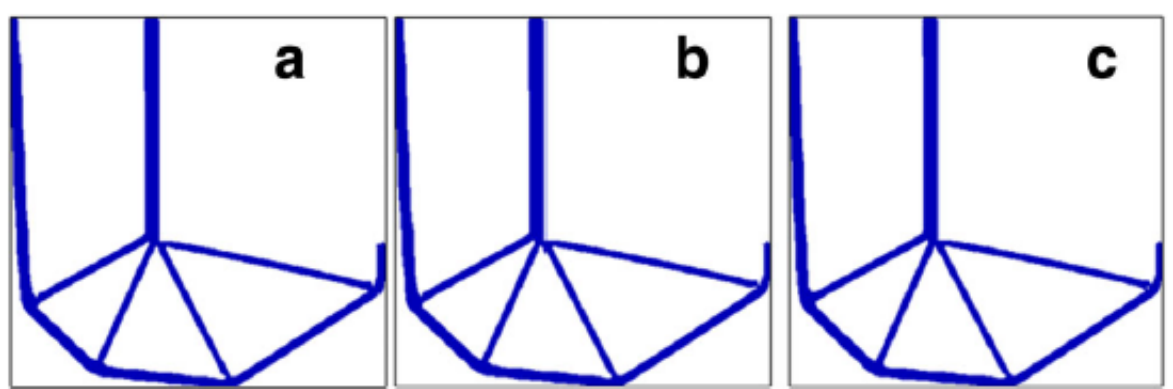

Figure 12. $L$ beam solutions with no microscale volume constraint a) uniform b) three c) 12 microstructures.

\subsection{Compliant mechanism design}

The multiscale topology optimization method is applied to design a compliant mechanism. The objective is to maximize the mechanical advantage, defined as the ratio of the output force, $F_{\text {out }}$, to the input force, $F_{\text {in }}$, subject to upper limits on the displacement at the input force location and the total volume (Sigmund 1997). The initial design, loading and boundary conditions are shown in Fig. 13, where only the lower half is modelled due to symmetry. The material properties are Young's modulus of ten and Poisson's ratio of 0.3 and the output stiffness, $k_{\text {out }}$, is 0.1 . The global volume constraint is $15 \%$ and the input displacement constraint is 20 . The macroscale domain is discretized using unit sized elements. The microscale unit cell is discretized into $50 \times 50$ elements and the initial design is the same as for the $\mathrm{L}$ beam example, Fig. $7 \mathrm{~b}$. The problem is solved with a different number of unique microstructures: one (uniform), 4, 16, and 1 microstructure per element, making a total of 7200 microstructures. The mappings for the 4 and 16 microstructure designs is shown in Fig. 14. All problems are solved without microscale volume constraints. For comparison, the problem is also solved using a single scale optimization using solid isotropic material.

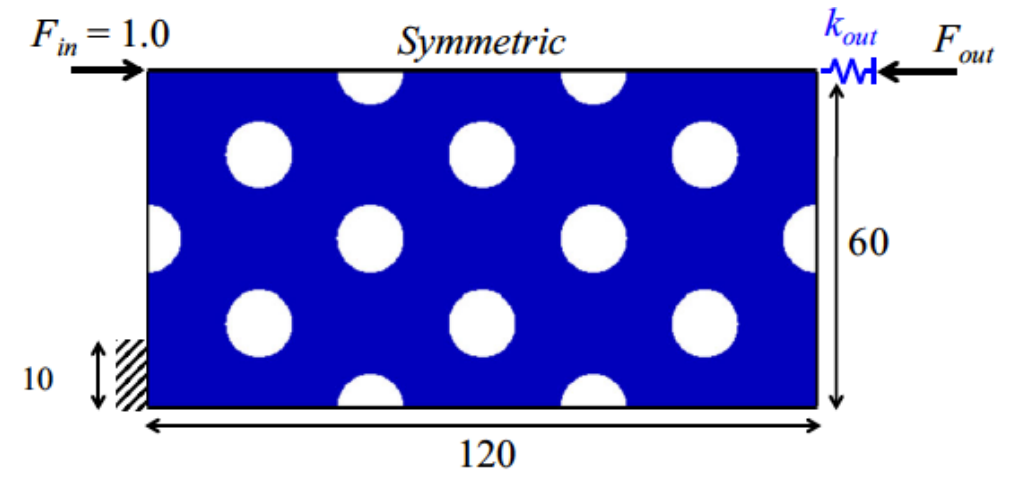

Figure 13. Compliant mechanism - Initial design, loading and boundary conditions.

(a)

\begin{tabular}{|l|l|}
\hline 3 & 4 \\
\hline 1 & 2 \\
\hline
\end{tabular}

(b)

\begin{tabular}{|c|c|c|c|}
\hline 11 & 12 & 15 & 16 \\
\hline 9 & 10 & 13 & 14 \\
\hline 3 & 4 & 7 & 8 \\
\hline 1 & 2 & 5 & 6 \\
\hline
\end{tabular}

Figure 14. Compliant Mechanism mapping of microstructures to macrostructure elements a) four microstructures b) 16 microstructures. 
The results show that as the number of unique microstructures increases, the mechanical advantage increases, Table 4. This again, demonstrates the expanded design space arising from material tailoring hence a better optimum solution can be found. However, unlike the previous compliance minimization example, this trend is seen without imposing microscale volume constraints and the multiscale optimization utilizes porous microstructure to improve the objective as compared with the solid material design. While the level-set method is applied at both macroscale and microscale, Figs. 15, 16, 17 and 18 show the plots of elemental porosity of the macroscale domain and the associated microstructures to show the topological solution and porosity of the structure. The results reflect how the optimizer makes use of porous microstructures to improve the objective.

The macroscale designs for the single scale and uniform microstructure solutions are similar, Fig. 15, and they are comparable with the results obtained by Bendsoe and Sigmund (2004). When nonuniform microstructures are allowed, porous microstructures aid in increasing the mechanical advantage compared with the solid material solution. Figures 16, 17 and 18 show that porous microstructures at some locations in the domain help in improving the objective as seen in Table 4. This suggests that the compliant mechanism is benefitting from the multiscale design. This may be explained because a compliant mechanism has conflicting stiffness requirements. It must be stiff enough to transmit the force from input to output locations and to meet the essential boundary conditions, but it must also be compliant to create the mechanism that changes the force direction at the output point.

When every element of the macroscopic domain is allowed to have its own microstructure, the optimizer has a significantly greater design space and more opportunities to tailor the material properties throughout the macroscopic structure. The local tailoring of the material microstructures near the input and output points leads to more compliant members. The porosity of the lower left member enables it to bend more than the solid microstructure counterpart. This leads to increase in objective function which further leads to the optimum solution. The advantage increased to 0.8901 .

Table 4. Compliant mechanism mechanical advantage values.

\begin{tabular}{|r|r|}
\hline $\begin{array}{c}\text { Number of } \\
\text { microstructures }\end{array}$ & $\begin{array}{c}\text { Mechanical } \\
\text { advantage }\end{array}$ \\
\hline single scale & 0.7403 \\
\hline 1 & 0.7461 \\
\hline 4 & 0.8522 \\
\hline 16 & 0.8644 \\
\hline 7,200 & 0.8901 \\
\hline
\end{tabular}




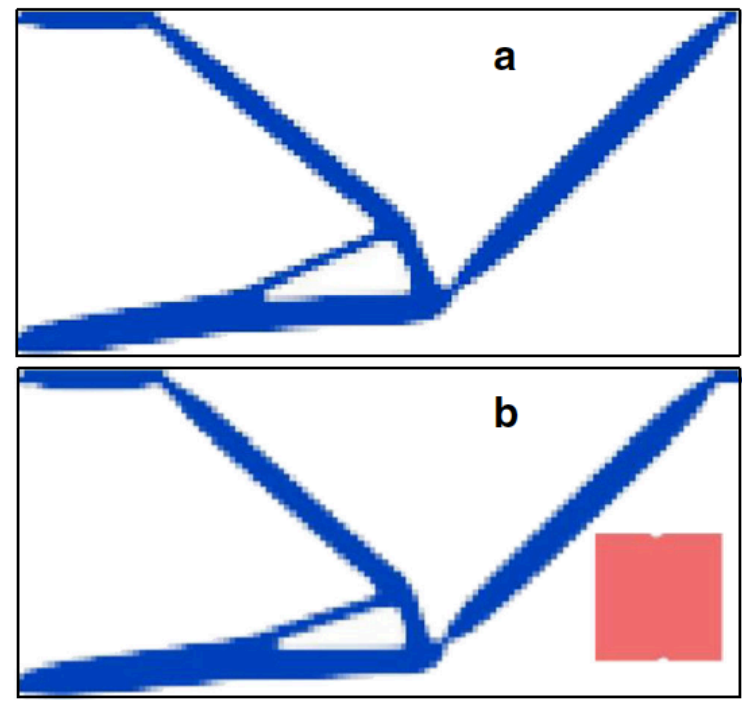

Figure 15. Elemental porosity plot of compliant mechanism - Optimum solution a) single scale b) uniform microstructure.

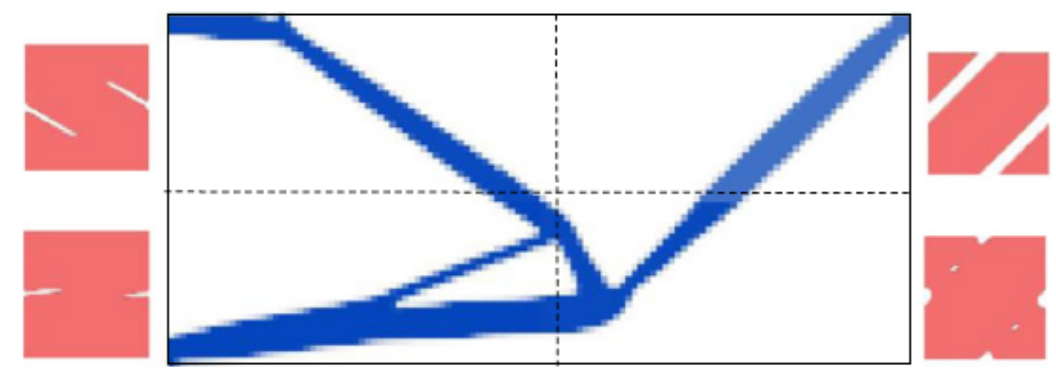

Figure 16. Elemental porosity plot of compliant mechanism - Optimum solution (four microstructures).

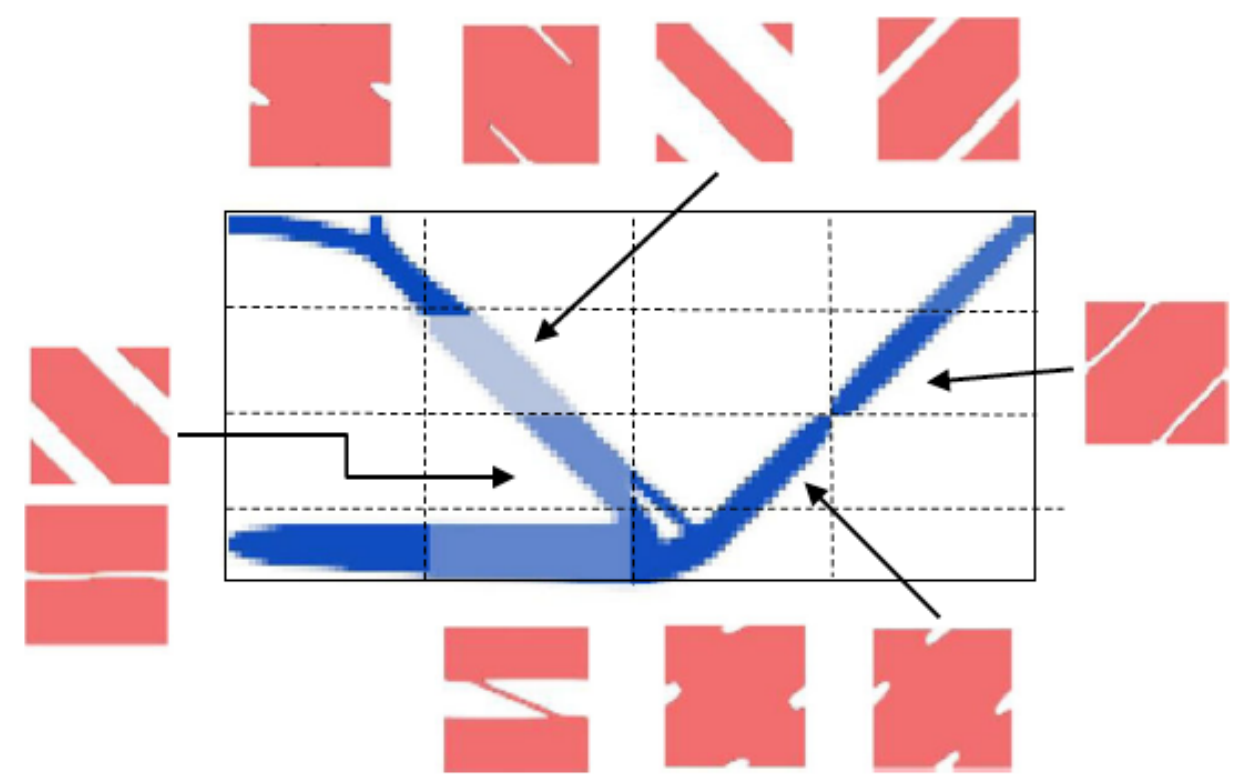

Figure 17. Elemental porosity plot of compliant mechanism - Optimum solution (12 microstructures). 


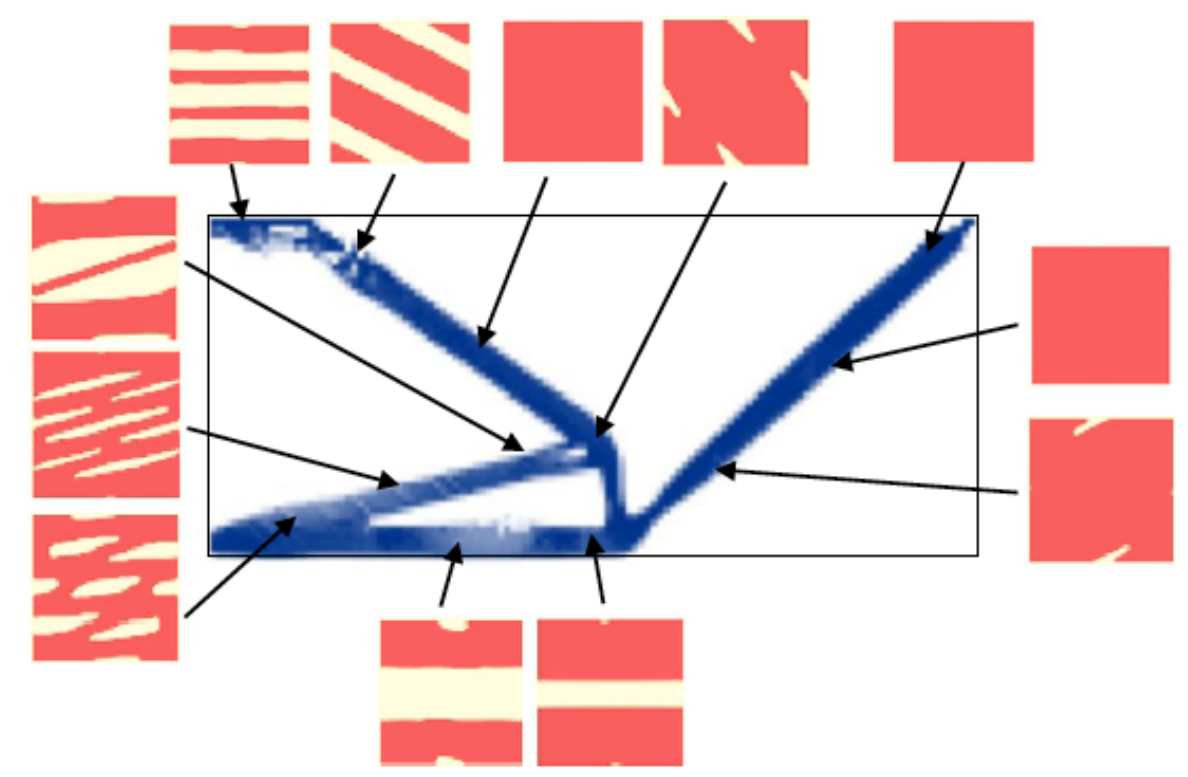

Figure 18. Elemental porosity plot of compliant mechanism - Optimum solution (One microstructure per element).

\section{Conclusions}

This paper introduces a new methodology for multiscale topology optimization, where two scales are linked by homogenization. The new method can optimize for any number of microscale domains, including uniform and nonuniform microscale designs. The decomposition formulation is proposed and this allows for easy parallelization of the solution algorithm. Thus, the computational time can be maintained at a practical level and the proposed methodology is scalable. The decomposition formulation results in several separate problems, one corresponding to the macroscopic domain and one for each of the microscale domains. The constraint boundaries for these problems are determined by formulating and solving it as an optimization problem.

The new multiscale topology optimization methodology is demonstrated using minimum compliance and compliant mechanism problems. The minimization of compliance problems from the existing literature are used to verify the methodology.

By switching off the macroscale topology optimization, the multiscale optimization reproduces the results of the classic topology optimization using homogenization. When topology optimization is applied for both macro and microscale domains, the optimum microscale design for minimum compliance with a single load case is found to be solid, and porous materials do not offer benefits. This means single scale optimization would provide the same topological solution as the multiscale solution for this problem. When microscale volume constraint is enforced, the objective improves as the number of allowable microstructures increase. This demonstrates that the multiscale optimization exploits the expanded design space offered by material tailoring. The porous materials is observed to be beneficial in compliant mechanism optimization where objective is improved by using a multiscale optimization as compared to a single scale optimization using solid isotropic material. When a uniform microstructure is used without microscale volume constraints, the resultant optimum solution is same as that obtained using a single scale optimization, and the optimum microstructure is solid. As the number of allowable microstructures increases, the objective improves reflecting the increased design space via local 
tailoring of material design. This demonstrates the potential benefits of multiscale topology optimization. Future work will study the effects of periodic boundary conditions at the interfaces of differing microstructures and at the edges.

\section{Acknowledgements}

The authors acknowledge the support from Engineering and Physical Sciences Research Council, grant number EP/ M002322/1. The authors would also like to thank Numerical Analysis Group at the Rutherford Appleton Laboratory for their FORTRAN HSL packages (HSL, a collection of Fortran codes for large-scale scientific computation. See http://www.hsl.rl.ac.uk/).

\section{References}

Allaire G (1993) Two-scale convergence and homogenization of periodic structures, School on homogenization. ICTP, Trieste

Allaire G, Jouve F, Toader AM (2004) Structural optimization using sensitivity analysis and a level-set method Journal of Computational Physics 194:363-393 doi:10.1016/j.jcp.2003.09.032

Bendsoe MP, Kikuchi N (1988) Generating optimal topologies in structural design using a homogenization method Computer Methods In Applied Mechanics and Engineering 71:197-224

BendsoeMP, Sigmund O (2004) Topology optimization: theory, methods and applications. Springer, Germany

Coelho PG, Fernandes PR, Guedes JM, Rodrigues HC (2008) A hierarchical model for concurrent material and topology optimisation of three-dimensional structures Struct Multidiscip O 35:107-115 doi:10.1007/S00158-007-0141-3

De Leon DM, de Souza CE, Fonseca JSO, da Silva RGA (2012) Aeroelastic tailoring using fiber orientation and topology optimization Struct Multidiscip O 46:663677 doi: $10.1007 / \mathrm{s} 00158-012-0790-8$

Deaton JD, Grandhi RV (2014) A survey of structural and multidisciplinary continuum topology optimization: post 2000. Struct Multidiscip O 49:1-38

Deng JD, Yan J, Cheng GD (2013) Multi-objective concurrent topology optimization of thermoelastic structures composed of homogeneous porous material Struct Multidiscip O 47:583-597 doi:10.1007/S00158-012-0849-6

Dunning PD, Brampton CJ, Kim HA (2015) Simultaneous optimisation of structural topology and material grading using level set method Materials Science and Technology 31:884-894 doi:10.1179/1743284715y.0000000022

Johnson SG (2014) The NLopt nonlinear-optimization package. http://abinitio.mit.edu/nlopt

Liu L, Yan J, Cheng GD (2008) Optimum structure with homogeneous optimum truss-like material Comput Struct 86:1417-1425 doi:10.1016/J.Compstruc.2007.04.030

Lund E (2009) Buckling topology optimization of laminated multi-material composite $\begin{array}{llll}\text { shell structures } & \text { Composite } & \text { Structures } & 91: 158-167\end{array}$ doi:10.1016/J.Compstruct.2009.04.046

Niu B, Yan J, Cheng G (2009) Optimum structure with homogeneous optimum cellular material for maximum fundamental frequency Struct Multidiscip O 39:115-132 doi:10.1007/s00158-008-0334-4 
Osher S, Sethian JA (1988) Fronts propagating with curvature dependent speed: algorithms based on Hamilton-Jacobi formulations. Journal of Computational Physics 79(1):12-49. doi:10.1016/0021-9991(88)90002-2

Peeters D, Baalen Dv, Abdalla M (2014) Combining Topology and Lamination Parameter Optimisation. In: 55th AIAA/ASMe/ASCE/AHS/SC Structures, Structural Dynamics, and Materials Conference. AIAA SciTech. American Institute of Aeronautics and Astronautics. doi:10.2514/6.2014-1372

Rodrigues H, Guedes JM, Bendsoe MP (2002) Hierarchical optimization of material and structure Struct Multidiscip O 24:1-10 doi:10.1007/s00158-002-0209-z

Sanchez-Palencia (1980) Non homogeneous media and vibration theory, lecture notes in physics. Springer, Berlin

Schury F, Stingl M, Wein F (2012) Efficient Two-Scale Optimization of Manufacturable Graded Structures Siam J Sci Comput 34:711-733 doi: $10.1137 / 110850335$

Sigmund O (1997) On the design of compliant mechanisms using topology optimization Mechanics of Structures and Machines 25:493-524

Sigmund O (2001) Design of multiphysics actuators using topology optimization Part II: Two-material structures Computer Methods in Applied Mechanics and Engineering 190:6605-6627 doi:10.1016/S0045-7825(01)00252-3

Sigmund O, Torquato S (1997) Design of materials with extreme thermal expansion using a three-phase topology optimization method J Mech Phys Solids 45:10371067 doi:10.1016/S0022-5096(96)00114-7

Wang MY, Wang X (2004) "Color" level sets: a multi-phase method for structural topology optimization with multiple materials Computer Methods In Applied Mechanics and Engineering 193:469-496 doi:10.1016/j.cma.2003.10.008

Wang MY, Wang X, Guo D (2003) A level set method for structural topology optimization Computer Methods In Applied Mechanics and Engineering 192:227-246

Xia L, Breitkopf P (2014) Concurrent topology optimization design of material and structure within nonlinear multiscale analysis framework Computer Methods in Applied Mechanics and Engineering 278:524-542 doi:10.1016/j.cma.2014.05.022

Yan X, Huang X, Zha Y, Xie YM (2014) Concurrent topology optimization of structures and their composite microstructures Comput Struct 133:103-110 doi:10.1016/J.Compstruc.2013.12.001 